\title{
Three-dimensional Segmentation and Quantification of the Anatomic Regurgitant Orifice in Mitral Regurgitation using 3D Ultrasound Images
}

\author{
Miguel Sotaquira ${ }^{1,3}$, Mauro Pepi ${ }^{2}$, Gloria Tamborini², Enrico Caiani ${ }^{3}$ \\ ${ }^{1}$ Universidad de San Buenaventura, Bogota, Colombia \\ ${ }^{2}$ Centro Cardiologico Monzino IRCCS, Milan, Italy \\ ${ }^{3}$ Politecnico di Milano, Dipartimento di Elettronica, Informazione e Bioingegneria, Milan, Italy
}

\begin{abstract}
Currently, clinical assessment of mitral regurgitation (MR) makes the use of 2D color Doppler imaging for the estimation of the vena contracta (VC) and of the effective regurgitant orifice area (EROA)..Since the anatomic regurgitant orifice (ARO) has a $3 D$ shape and it's not circular, it cannot be accurately represented by these $2 D$ parameters. We developed a novel semiautomated method for 3D ARO segmentation and quantification using $3 D$ transesophageal echocardiographic (TEE) datasets, and validated it vs manual planimetry on a set of 25 patients with mild to severe MR. ARO $2 D$ projected area and circularity index (CI) correlated well with planimetry results $\left(r^{2}=0.77\right.$ and 0.90 and bias of -0.02 and 0.02, respectively). In 19/25 patients ARO planarity index was less than 0.9, confirming its $3 D$ morphology. Only 1/25 patients exhibited an almost circular ARO (CI > 0.92), while 24/25 patients had more elongated orifices $(C I<0.8)$.

In conclusion, volumetric quantification of $3 D$ ARO is feasible, and the results confirm the potential of this technique for the estimation of real $3 D$ ARO shape. The proposed method could be a useful alternative for the assessment of patients with MR, given the intrinsic shape of the ARO and the limitations present in current approaches.
\end{abstract}

\section{Introduction}

Current guidelines for the quantitative assessment of mitral regurgitation (MR) recommend the use of twodimensional (2D) color Doppler flow imaging for the estimation of the vena contracta (VC), the effective regurgitant orifice area (EROA) and the regurgitant volume[1]. To require careful setting of the acquisition parameters, these 2D-based approaches rely on the assumption that the regurgitant orifice lies on a plane and is almost circular, a requirement that is not satisfied in many MR pathologies given the intrinsic 3D morphology of the orifice[2].

We hypothesized that the information contained in the 3D transesophageal echocardiographic (TEE) dataset, along with the use of advanced image processing and computational geometry algorithms, should allow the detection and quantification of the 3D anatomic regurgitant orifice (ARO) shape. Accordingly, our aims were: 1) to develop a nearly-automated algorithm for ARO segmentation from 3D TEE images; 2) to extract the $3 \mathrm{D}$ surface representing the ARO, and to compute several morphological parameters from this representation; 3) to validate the accuracy of the detection by applying manual planimetry of the orifice in patients with MR; and 4) to characterize the morphology of the orifice both in patients with degenerative mitral valve (MV) disease and in functional MR.

\section{Methods}

\subsection{Population and imaging protocol}

Twenty-five patients (age $63 \pm 8$ years) with mild to severe MR undergoing clinically indicated 3D TEE at Centro Cardiologico Monzino (Milan, Italy) were retrospectively selected. Of these 25 patients, 12 had functional MR (FMR group), whereas the remaining 13 patients presented MV prolapse (MVP group), with MR resulting from degenerative MV disease.

Images were acquired using the iE33 system (Philips Medical Systems, Andover, MA) equipped with fully sampled matrix-array 3D TEE transducer (X7-2t), with each acquired volume including the MV, the aortic valve and the proximal ascending aorta, and having dimensions of roughly 200x200x200 voxels with a voxel resolution ranging from 0.2 to $1.3 \mathrm{~mm}$. 


\subsection{Image analysis}

Datasets were analysed using both freeware and custom software. After selecting a systolic frame from the 3D TEE dataset, user-guided 3D segmentation of the region containing the orifice and some portions of the atrial and ventricular cavities was performed, thus ensuring that the segmented structures will closely follow the shape of the regurgitant jet. This initial step, performed using ITK-Snap, uses an active contour segmentation approach[3], and requires only one userdefined point located near the regurgitant orifice (Figure 1a). Next, the polygonal 3D manifold mesh representing the segmented structure is exported for further processing in Matlab. This mesh consists of two concave shapes (corresponding to the partial segmentation of atrium and ventricle) connected by a narrow structure, or channel, corresponding to the regurgitant orifice (Figure $1 \mathrm{~b}$ ).

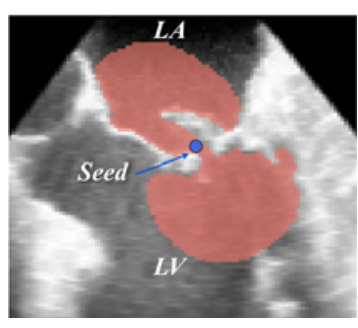

(a)

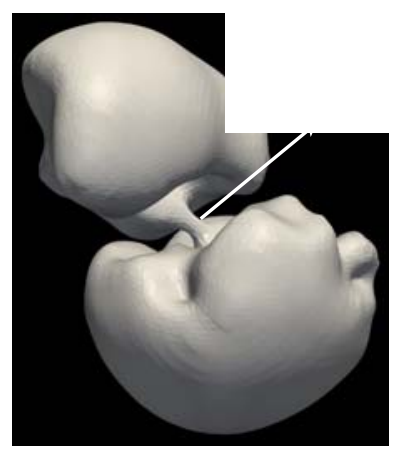

(b)
Figure 1. Initial segmentation of the region containing the ARO: (a) 2D cut-plane depicting the location of the user-defined seed (blue) and the segmented region containing the ARO (red); (b) 3D mesh representing the segmented structure, and the channel containing the ARO. LA: left atrium; LV: left ventricle.

The goal is then to automatically delineate the 3D contour of the narrowest portion of this channel, corresponding to the ARO. To do so, two shape descriptors are computed for each vertex on the $3 \mathrm{D}$ mesh: a shape diameter function[4] and the average geodesic distance [5]. These descriptors constitute the inputs of a max-flow/min-cut graph algorithm[6] which, when applied on a directed graph defined by the mesh's vertices, automatically finds the 3D ARO's contour (Figure 2).

After smoothing the computed contour, to minimize the noise present in both the original 3D TEE image and in the computed mesh, a thin-plate spline interpolation is applied to obtain the 3D mesh representation of the ARO and its 2D projected surface (Figure 3a). From these representations, several morphologic parameters describing ARO's shape were defined:

(1) 3D and 2D areas

(2) Planarity index, defined as the ratio between 2D and 3D ARO areas (values closer to 1 denote more planar orifices)

(3) Circularity index, defined as the ratio between the major and minor axes of the ellipse fitting the 2D representation of the orifice (values closer to 1 denote more circular orifices).

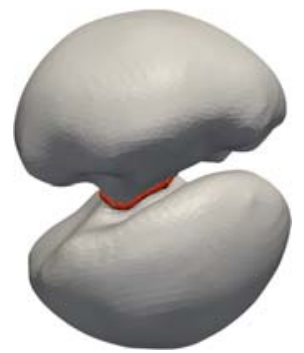

(a)

Figure 2. Original 3D mesh (gray) and the corresponding segmented ARO 3D contour (red) obtained using the max-flow/min-cut algorithm.

\subsection{Validation protocol}

Linear correlation and Bland-Altman analyses were applied to compare 2D ARO area and circularity index with the corresponding parameters obtained from manual planimetry (Figure 3) performed by an expert on the original 3D TEE datasets, blinded to the results of segmentation. Comparisons were performed for the entire group of $25 \mathrm{MR}$ patients.

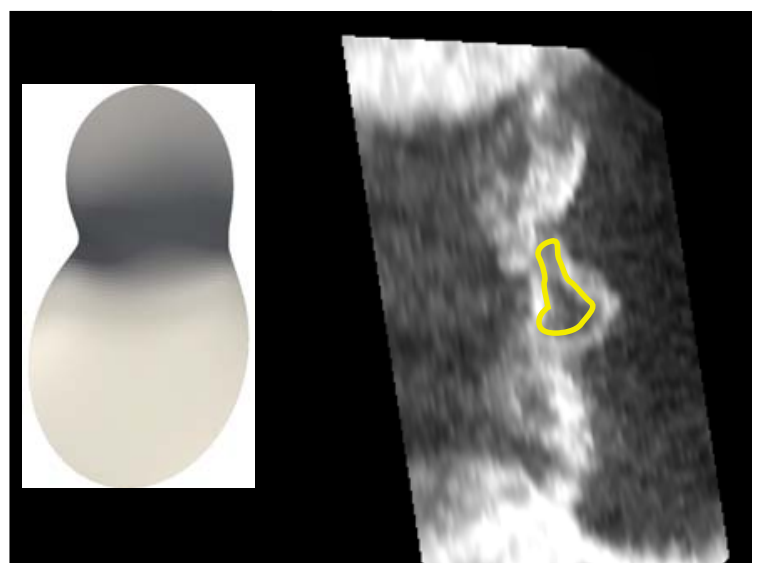

(a)

(b)

Figure 3. Examples of: (a) 2D projection of the computed 3D ARO mesh; and (b) Manual ARO planimetry. 
Table 1. Bland-Altman and linear regression results of ARO 2D area and circularity index on the studied population $(n=25)$. CI: confidence intervals for Bland- Altman analysis; $\mathrm{m}$ and $\mathrm{b}$ : linear regression coefficients; $r^{2}$ : coefficient of determination.

\begin{tabular}{l|cc|ccc}
\hline \multirow{2}{*}{\multicolumn{1}{c|}{ Parameter }} & \multicolumn{2}{|c|}{ Bland-Altman } & \multicolumn{3}{c}{ Linear regression } \\
\cline { 2 - 6 } & \multicolumn{1}{c}{ Bias } & $C I$ & $m$ & $b$ & $r^{2}$ \\
\hline 2D area $\left(\mathrm{cm}^{2}\right)$ & -0.02 & \pm 0.15 & 1.15 & -0.01 & 0.77 \\
Circularity index (a.i) & 0.02 & \pm 0.13 & 0.89 & 0.04 & 0.90 \\
\hline
\end{tabular}

Table 2. Comparison of computed morphological parameters, both in functional MR (FMR) and mitral valve prolapse (MVP).

\begin{tabular}{l|l|l}
\hline \hline \multicolumn{1}{c|}{ Group } & \multicolumn{1}{c|}{ FMR } & \multicolumn{1}{c}{ MVP } \\
Parameter & & \\
\hline 3D ARO surface $\left(\mathrm{cm}^{2}\right)$ & $0.13(0.09 ; 0.42)$ & $0.30(0.17 ; 0.33)$ \\
2D ARO area $\left(\mathrm{cm}^{2}\right)$ & $0.12(0.08 ; 0.38)^{\xi}$ & $0.17(0.13 ; 0.27)^{\xi}$ \\
Planarity index (a.u.) & $0.90(0.84 ; 0.92)$ & $0.77(0.66 ; 0.78)^{\gamma}$ \\
Circularity (a.u.) & $0.62(0.36 ; 0.68)$ & $0.56(0.50 ; 0.60)$ \\
\hline${ }^{\xi} \mathrm{p}<0.01$ 2D vs. 3D ARO surface area, Student’s paired t-test \\
${ }^{\gamma} \mathrm{p}<0.01$ vs. FMR, Mann-Whitney U test
\end{tabular}

\subsection{ARO morphology in different populations}

Each group of patients was also assessed in terms of the computed parameters of the 3D ARO. To test for differences between FMR and MVP groups, the MannWhitney U test was applied. To test for differences between the 2D and 3D ARO areas, within the FMR and MVP groups, the Student's paired t-test was applied. In all tests, differences were considered significant for $\mathrm{p}<0.01$.

\section{Results}

Table 1 summarizes the results of the linear correlation and Bland-Altman analyses performed when comparing our method against 2D ARO area and circularity index obtained using manual planimetry. Very small biases and narrow limits of agreement were observed in the studied population for both parameters, where the circularity index exhibited a higher determination coefficient than $2 \mathrm{D}$ area (0.90 vs 0.77$)$.

Table 2 lists the median, 25\% and 75\% percentiles of the 3D and 2D ARO areas, as well as the circularity and planarity indices on each population. 3D ARO surface area was not different between FMR and MVP, while 2D ARO projected area was underestimating 3D ARO surface area in both groups (43\% in the MVP, and 7.7\% in the FMR group). As expected from pathophysiology [2], the FMR group exhibited a more planar ARO than in MVP (0.90 vs 0.77, respectively). In the combined groups, 19 out of 25 patients exhibited planarity index less than 0.9, thus confirming the 3D non-planar ARO morphology. Both groups showed elongated AROs: only one patient exhibited an almost circular ARO (circularity index of 0.92), while the remaining 24 had more elongated ARO (circularity index less than 0.8 ).

\section{Discussion}

The proposed method demonstrated that ARO segmentation and quantification from 3D TEE datasets is feasible in patients with different MR etiologies. Our segmentation strategy requires minimal user interaction (only one seed point defined by the user), and is able of automatically delineating the 3D ARO contour and characterizing morphologically the detected orifice. A main advantage of the proposed approach is that it relies on the information contained in the grayscale 3D TEE dataset, thus eliminating the need of 3D color Doppler information, which in turn would decrease frame rate in the acquisition and would require additional steps in the analysis.

When compared with other methods, the proposed procedure also overcomes previous limitations: (i) it requires minimal user intervention, in contrast to Chandra et al[7] requiring manual tracing of mitral leaflets on multiple 2D cut-planes; (ii) it requires only one systolic 3D TEE frame, whereas Moraldo et al[8] relies on assumptions about the circularity of the orifice and requires multiple systolic frames to obtain an approximation to the ARO size; (iii) it makes use of existent technologies for the acquisition of 3D TEE datasets, with no need to acquire 3D color Doppler images, as proposed by Grady et al[9]; and finally (iv) the method doesn't make any assumptions about the shape of the orifice (its circularity and planarity), which is the basis of current clinical standards for the estimation of EROA and VC.

Validation with manual planimetry for 2D projected 
area and circularity showed a very good agreement of the automatically obtained measurements, with higher agreement for the circularity index. This can be explained by the fact that this index represents a ratio between the minor and major axes of the 2D ARO. An overestimation of the ARO area, resulting from an increased length in both axes, is masked by the ratio computation.

From the comparisons, statistically significant differences became evident. First, the computed 2D areas differed from the 3D ARO surfaces in both groups, which can be explained by the intrinsic 3D morphology of the regurgitant orifice, not fully captured by a planar index. This result stresses the limitations of current 2D-color Doppler based approaches (EROA and $\mathrm{VC}$ estimation) for the assessment of patients with MR. Additionally, by comparing MVP vs FMR, an expected difference in planarity was found, with higher values associated to FMR.

Second, most of the patients included in this study (24/25) exhibited elongated, rather than almost circular, regurgitant orifices (with MVP showing a trend in presenting lower circularity index when compared to FMR).

As previously mentioned, the observed non-planarity and non-circularity of the regurgitant orifice stress the limitations of current clinical standards used for the assessment of mitral regurgitation. Since they rely on the spatial location of the corresponding 2D cut-plane, EROA and VC computation tend to over/under-estimate the real size of the regurgitant orifice, which can have profound implications in classification of MR severity. On the contrary, the method here proposed works directly on the 3D dataset, and thus the computed ARO is a more reliable approximation of the true orifice's shape, not related to a particular 2D cut-plane, which in turn may lead to a more accurate assessment of patients with MR.

\subsection{Limitations}

The main limitation of the proposed technique is constituted by the lack of a true "gold standard" for the geometry of the regurgitant orifice. However, we tried to overcome it by manually obtaining the contours of the regurgitant orifice by careful selection of the proper cut-plane.

\section{Conclusions}

We proposed and preliminary validated a novel method for the segmentation and quantification of the 3D ARO from 3D TEE images in patients with MR.

The method reliably estimates the real ARO shape, as shown by the good levels of correlation, small biases and narrow limits of agreement when compared with manual planimetry. In addition, our results call into question the use of EROA and VC approaches for the assessment of MR severity, since these methods rely on strong assumptions about the planarity and circularity of the orifice, assumptions that were not verified in most of the patients within the studied population.

\section{References}

[1] Zoghbi WA, Enriquez-Sarano M, Foster E, Grayburn P, Kraft C, et al. Recommendations for evaluation of the severity of native valvular regurgitation with twodimensional and doppler echocardiography. Journal of the American Society of Echocardiography (2003); 16: 777802.

[2] Little SH, Igo SR, Pirat B, McCulloch M, Hartley CJ, et al. In Vitro Validation of Real-Time Three-Dimensional Color Doppler Echocardiography for Direct Measurement of Proximal Isovelocity Surface Area in Mitral Regurgitation. The American Journal of Cardiology (2007); 99: 1440-1447.

[3] Zhu SC, Yuille A. Region Competition: Unifying Snakes, Region Growing and Bayes/MDL for Multiband Image Segmentation. IEEE Transactions on Pattern Analysis and Machine Intelligence (1996); 18: 884-900.

[4] Shapira L, Shamir A, Cohen-Or D. Consistent mesh partitioning and skeletonisation using the shape diameter function. Visual Computing (2008); 24: 249-259.

[5] Zhang E, Mischaikow K. Feature-Based Surface Parameterization and Texture Mapping. ACM Transactions On Graphics (2005); 24, 1-27.

[6] Boykov Y, Kolmogorov V. An experimental comparison of min-cut/max-flow algorithms for energy minimization in vision. IEEE Transactions on Pattern Analysis and Machine Intelligence (2001); 26, 359-374.

[7] Chandra S, Salgo IS, Sugeng L, Weinert L, Settlemier SH, et al. A three-dimensional insight into the complexity of flow convergence in mitral regurgitation: adjunctive benefit of anatomic regurgitant orifice area. AJP: Heart and Circulatory Physiology (2011); 301, H1015-H1024.

[8] Moraldo M, Bergamini C, Malaweera ASN, Dhutia NM, Pabari PA, et al. A novel fully automated method for mitral regurgitant orifice area quantification. International Journal of Cardiology (2013); 166, 688-695.

[9] Grady L; Datta S, Kutter O, Duong C. Regurgitation quantification using 3D PISA in volume echocardiography. Proceedings Medical Image Computing and Computer-Assisted Intervention MICCAI 2011 (2011); 512-519.

Address for correspondence.

Miguel Sotaquira, $\mathrm{PhD}$

Faculty of Engineering, Universidad de San Buenaventura Carrera 8H \# 172-20

Bogota, Colombia

E-mail: msotaquira@usbbog.edu.co 